\title{
A Framework to Analyze Data on Homelessness, Poverty and Migration Using Fuzzy Cognitive Modeling
}

Naeem, R.*; Walford, N.**; Kauppi, C.*; Pallard, H.*

*Laurentian University, Sudbury, Ontario, Canada

**Kingston University, Kingston, UK

Rahat Naeem: naeem@.clickmox.com

Nigel Walford: nwalford@kingston.ac.uk

Carol Kauppi: ckauppi@,laurentian.ca

Henri Pallard: hpallard@laurentian.ca 


\title{
A Framework to Analyze Data on Homelessness, Poverty and Migration Using Fuzzy Cognitive Modeling
}

\begin{abstract}
This article develops a framework for studying homelessness, poverty and migration using Fuzzy Cognitive Modeling. The framework has been developed using data obtained from a survey of around 4000 respondents in five communities in northeastern Ontario, Canada. The intention was to develop a framework that could be applied in other communities where comparable sources of data are available. This framework can be used to perform sensitivity analysis as well as to develop index of poverty as discussed in this paper. The results suggest that this method provides a robust approach to analyze complex and multivariate datasets related to homelessness, poverty and migration with variables that not only have interdependencies but are also not crisp.
\end{abstract}

Keywords: Homelessness, poverty, migration, fuzzy cognitive mapping, fuzzy cognitive model, poverty index

\section{Introduction}

Homelessness and poverty are complex phenomena and depend on many variables that themselves have interdependencies (Fowler et.al. 2019). A Fuzzy Cognitive Model (FCM) provides an excellent framework to perform multivariate analysis on complex datasets (Dickerson and Kosko, 1994). Although the technique is now being implemented in many different disciplines, it was originally developed to analyze social models. This was due to the fact that in social datasets the logic is generally not crisp. For example, poverty and 
homelessness cannot be defined by crisp logic due to the complexity of the issues. Hence fuzzy logic is of central importance in building FCM. An FCM is constructed in the form of the so called Fuzzy Cognitive Map (also referred to as FCM), which in simple terms is the combination of fuzzy logic and cognitive mapping. Figure 1 shows a typical FCM consisting of four variables (A, B, C, D), called nodes. The arrows between the nodes and the associated numbers show the strengths of the relationships between them (Gray, 2013). These strengths, called fuzzy weights, are based on fuzzy logic and are assigned with the help of an assignment table. This assignment table is generally constructed by analyzing the dataset. The direction of the arrow shows whether the relationship between the variables is positive or negative.

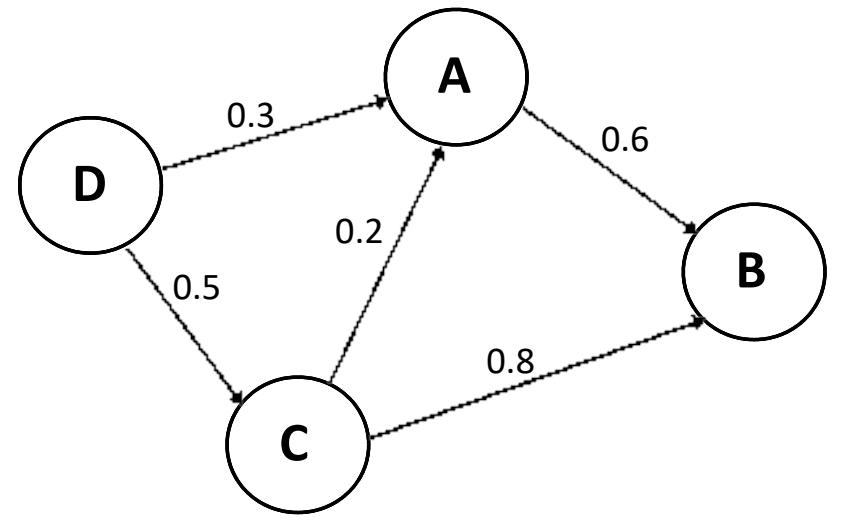

Figure 1: A typical Fuzzy Cognitive Map.

Once the nodes and their interconnected fuzzy weights have been established, the influences of the nodes on each other are calculated using an iterative procedure based on a neural network approach. Once a stable solution has been obtained, the model can be used to determine the behaviour of the system as node values are changed. This is what is termed the sensitivity analysis. In this paper we describe a framework that has been developed to analyze data related to poverty, homelessness and migration using the FCM approach. The data used for this development was gathered within a broader study - Poverty Homelessness and Migration (PHM) - performed at 
Laurentian University, Canada and analyzed as part of the $\mathrm{PhD}$ research conducted by the lead author of this paper (Naeem, 2020).

\section{Fuzzy Cognitive Framework}

In this study the following variables were initially used to generate the FCMs.

Access to Education - Access to Health - Age - Domestic Violence - Education Level Employment Status - Ethnicity - Family Problems - Gender - Marital Status - Mental Health Migration - Number of Children - Physical Health - Substance Abuse.

It should be noted that an FCM based on all these variables is very complex and difficult to draw inferences from. The situation is further complicated by the fact that the data collected from different study communities revealed different interdependencies of variables. This was expected since distributions of the variables had already hinted towards this issue. For example, the age distributions of homeless individuals in different regions showed some differences. It was therefore necessary to develop an FCM for each study community and data collection period separately. In order to develop a framework to understand effects of different variables on homelessness, it was necessary to simplify these fuzzy cognitive models so that a common framework for comparison could be developed. It is worth noting that some of the above-mentioned variables are likely to have negligible impact on homelessness. However, instead of making a subjective decision, data

analyses on all these variables were performed to determine which ones were most important with regard to development of simplified fuzzy cognitive maps. 
As discussed above, the fuzzy relationships (or weights) in a fuzzy cognitive map are not necessarily crisp and should be deduced from the data collected during the study. In the context of fuzzy logic, a relationship can be positive or negative and can have any value between -1 and +1 . For this study, the relationship levels, as shown in Table 1, were initially used since the FCM MentalModeler software available at that time only supported six values of logic levels (Gray, 2013). Subsequently, an update of the software became available that did not have this restriction and was thus used to complete the analyses.

Table 1: Fuzzy Logic Levels

\begin{tabular}{|c|c|}
\hline Effect & Value \\
\hline $\mathrm{H}+$ & 1 \\
\hline $\mathrm{M}+$ & 0.5 \\
\hline $\mathrm{L}+$ & 0.25 \\
\hline $\mathrm{L}-$ & -0.25 \\
\hline $\mathrm{M}-$ & -0.5 \\
\hline $\mathrm{H}-$ & -1 \\
\hline
\end{tabular}

In order to deduce the weight values from the data collected, filter and statistics functions available in SPSS were used. In the main database (SPSS) some of the variables were assigned numerical values, such as 1 for female and 2 for male. Variables such as age assumed the actual values while those that were measured using yes or no answers were assigned a numerical value (e.g. 1 and 2). An important step in analyses was to determine interdependence of these variables so that an FCM could be constructed. An efficient way to determine such interdependencies is to calculate the Pearson's correlation coefficient, which assumes that the relationship between the variables is linear (Hall, G. 2015). This is a valid assumption since the range of values the variables assume is small and no strong non-linearities are expected within that range. As mentioned above, the earlier version of the MentalModeler software used to generate FCMs accepted only six discrete values of the fuzzy weights as 
given in Table 1. This meant that, to deduce the fuzzy weights, the correlation coefficients must be mapped onto the fuzzy logic weights as listed in Table 1.

Table 2: Variable values as defined in SPSS.

\begin{tabular}{|c|c|}
\hline Variable & Assigned Values \\
\hline Access to Education & A unique value \\
\hline Access to Health & A unique value \\
\hline Age & Actual numerical values \\
\hline Domestic Violence & A unique value \\
\hline Education Level & Less than high school $=1, \ldots \ldots .$, University $=7$ \\
\hline Ethnicity & Caucasian $=1$, Indigenous $=2$ \\
\hline Family Problems & A unique value \\
\hline Gender & Female $=1$, Male $=2$ \\
\hline Homeless & $\mathrm{Yes}=1, \mathrm{No}=2$ \\
\hline Marital Status & Married/Common Law $=1$, Single/Divorced/Widowed $=2$ \\
\hline Mental Health Problems & $\mathrm{Yes}=1, \mathrm{No}=2$ \\
\hline Migration & $\mathrm{Yes}=1, \mathrm{No}=2$ \\
\hline Number of Children & Actual numerical values \\
\hline Physical Health Problems & $\mathrm{Yes}=1, \mathrm{No}=2$ \\
\hline Substance Abuse & A unique value \\
\hline
\end{tabular}

In the theory of statistics, it is known that the correlation between two variables can be characterized by the following scheme (see, for example, Hall, G 2015 and references therein).

Weak positive correlation: $\quad 0<r \leq 0.3$

Moderate positive correlation: $0.3<r \leq 0.5$

Strong positive correlation: $\quad 0.5<r \leq 1.0$

This correlation scheme is symmetric on the negative side. Using this, the correlation values were mapped on to the fuzzy logic levels as given in Table 3 below. Again, it should be noted that 
during the later stages of the analyses, the continuous fuzzy levels were used, which allowed direct 1:1 correspondence with the correlation coefficients.

Table 3: Fuzzy Logic Levels.

\begin{tabular}{|c|c|c|}
\hline Correlation Coefficient & Effect & Value \\
\hline $0.5<r \leq 1.0$ & $\mathrm{H}^{+}$ & 1 \\
\hline $0.3<r \leq 0.5$ & $\mathrm{M}+$ & 0.5 \\
\hline $0<r \leq 0.3$ & $\mathrm{~L}+$ & 0.25 \\
\hline$-0.3 \leq r<0$ & $\mathrm{~L}-$ & -0.25 \\
\hline$-0.5 \leq r<-0.3$ & $\mathrm{M}-$ & -0.5 \\
\hline$-1.0 \leq r<-0.5$ & $\mathrm{H}-$ & -1 \\
\hline
\end{tabular}

A typical fuzzy weight matrix generated in this way is shown in Table 4 below.

Table 4: Fuzzy weight matrix for data collected in Sudbury in year 2009.

\begin{tabular}{|c|c|c|c|c|c|c|c|c|c|c|}
\hline Sudbury 2009 & $\begin{array}{l}\dot{\bar{\theta}} \\
\bar{D} \\
0 \\
0\end{array}$ & $\stackrel{8}{\&}$ & 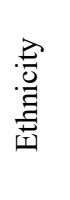 & 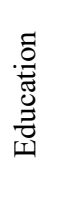 & $\begin{array}{l}\text { 节 } \\
\text { 总 } \\
\text { 言 }\end{array}$ & 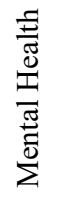 & 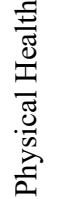 & $\begin{array}{l}\frac{y}{0} \\
\frac{1}{0} \\
\dot{0} \\
\dot{z}\end{array}$ & 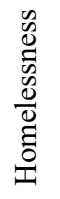 & 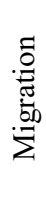 \\
\hline \multicolumn{11}{|l|}{ Gender } \\
\hline Age & $\mathrm{L}+$ & & & & & & & & & \\
\hline Ethnicity & $\mathrm{L}+$ & L- & & & & & & & & \\
\hline Education & L- & L- & L- & & & & & & & \\
\hline Employment & L- & L- & L- & L- & & & & & & \\
\hline Mental Health & $\mathrm{L}+$ & L- & L- & L- & L- & & & & & \\
\hline Physical Health & $\mathrm{L}+$ & M- & L- & L- & L- & $\mathrm{M}+$ & & & & \\
\hline No. of Kids & L- & $\mathrm{L}+$ & $\mathrm{L}+$ & L- & L- & L- & L- & & & \\
\hline Homelessness & $\mathrm{L}+$ & $\mathrm{L}+$ & L- & L- & L- & $\mathrm{L}+$ & $\mathrm{L}+$ & L- & & \\
\hline Migration & $\mathrm{L}+$ & $\mathrm{L}+$ & $\mathrm{L}+$ & L- & L- & L- & L- & L- & L- & \\
\hline
\end{tabular}


The FCM is constructed by first building relationship map between all variables and then assigning weights to those variables in the weight value matrix. In order to determine the weights, distributions of different variables were generated. For example, consider the interplay between eviction and gender in Sudbury between the years 2000 and 2009 as depicted in Table 5 below.

Table 5: Effect of eviction on gender in Sudbury between the years 2000 and 2009.

\begin{tabular}{|l|r|c|c|c|c|}
\hline Location & Study Year & Female (\%) & Male (\%) & $\begin{array}{c}\text { Transgender } \\
\mathbf{( \% )}\end{array}$ & Subjects \\
\hline Sudbury & 2001 & 44.4 & 55.6 & & 27 \\
\hline Sudbury & 2002 & 20 & 80 & & 40 \\
\hline Sudbury & 2003 & 34.8 & 65.2 & & 69 \\
\hline Sudbury & 2007 & 40.8 & 59.2 & & 76 \\
\hline Sudbury & 2009 & 43.2 & 55.4 & 1.4 & 74 \\
\hline
\end{tabular}

To determine the FCM weight from this table, it should first be noted that females and males were assigned valued of 1 and 2 respectively. It should be mentioned that this assignment does not have any effect on the overall FCM analysis since if the assignment is reversed it will be reversed for all the factors. Keeping this assignment in view, it was noted that in year 2001, there was only a slight direct correlation between gender and eviction rate and therefore an FCM weight of 0.25 or L + can be assigned. On the other hand, in year 2002, there seems to be a very strong bias in eviction rate for males and therefore an FCM weight of +1 or $\mathrm{H}+$ should be assigned.

Once all the weights have been assigned, the FCM map was generated with the help of the Mental Modeler software package. Figure 2 shows a typical FCM map generated during this study. 


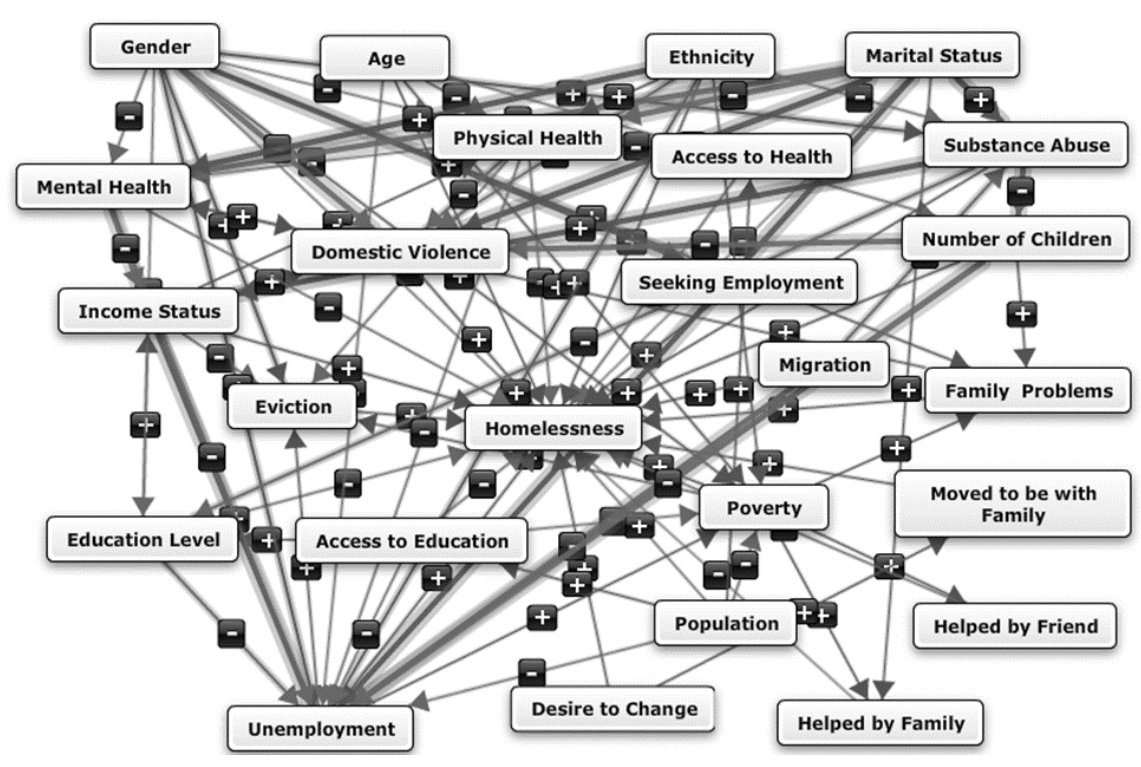

Figure 2: Typical Fuzzy Cognitive Map generated during this study.

The thickness of the interconnections represents the strength of correlation (positive or negative) between the variables. This FCM is very complex due to inter-relationships between the variables and therefore very difficult to use and interpret. In order to develop a simplified FCM for homelessness, sensitivity analyses were done on this model. To do this, artificial scenarios were created, such as increase in overall mental health of the population. Running the scenario showed its effect on different parameters. Figure 3 shows a typical scenario chart. 


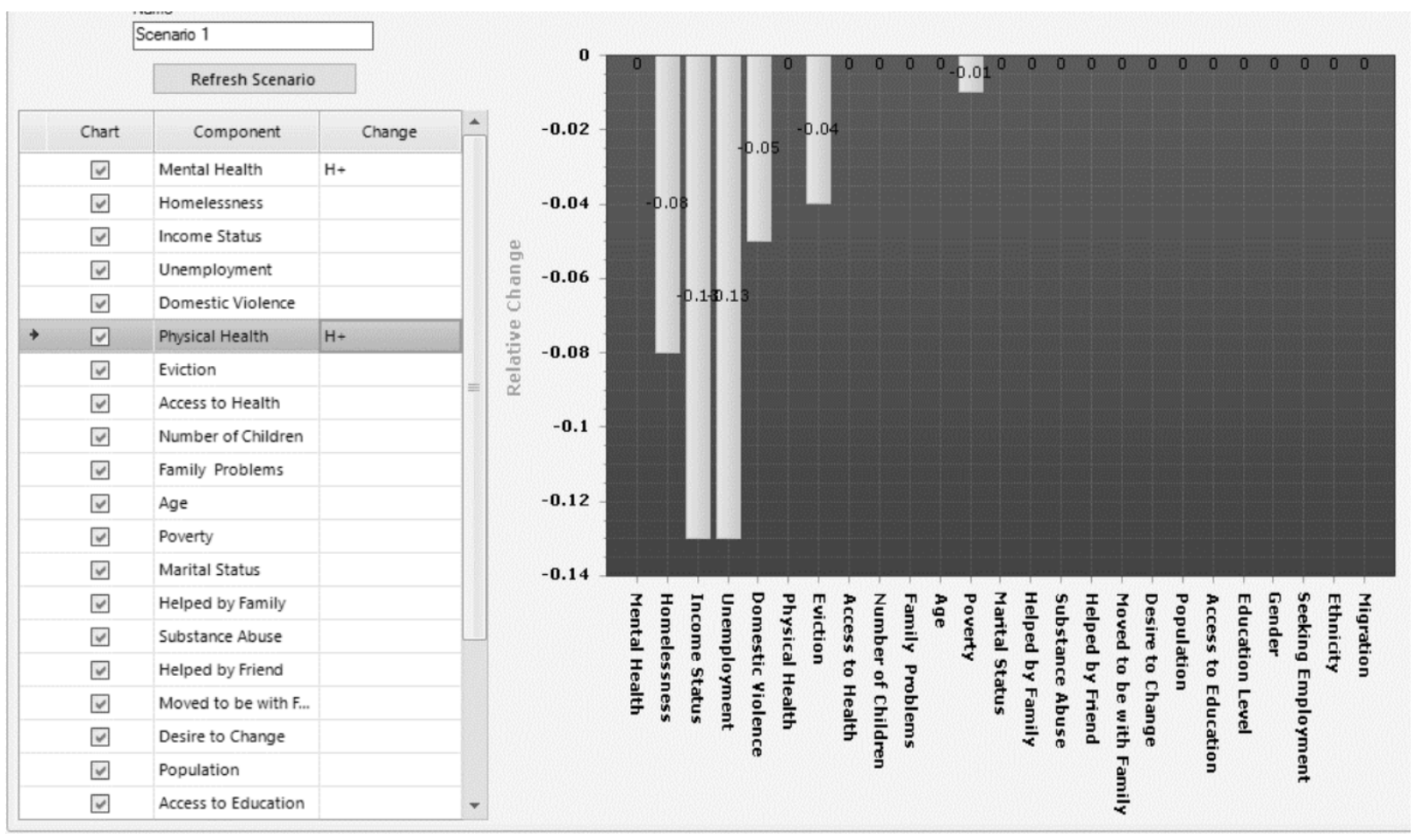

Figure 3: Typical FCM scenario chart.

In this scenario, the mental and physical health of the population was increased to the highest possible value $(\mathrm{H}+)$. This has reduced the homelessness by $8 \%$ and unemployment by $13 \%$. Repeating this process for all six values for physical health from $\mathrm{H}+$ to $\mathrm{H}$ - gave six values for change in unemployment. The relationship between the fuzzy levels and the corresponding change in homelessness gave a measure of direct relationship between that particular variable and homelessness. However, this gave six values, which means the relationship is not crisp and should therefore be handled in the framework of fuzzy logic. In order to get one value, these values were de-fuzzified using the centroid method (Wang, 2009) as explained below.

The first step is to normalize the values obtained from the above analysis. This can be performed by denoting the fuzzy value of the $i$ th attribute and the $j$ th value by $x_{i}^{J}$. Then the normalized fuzzy value for the $i$ th attribute is calculated as follows: 


$$
x_{i}=\frac{x_{i}^{J}}{\sum_{j=1}^{6} x_{i}^{j}}
$$

All the $x_{i}$ values thus obtained together form the so-called fuzzy membership function $\mu(z)$ with $z$ being the values at which the $x_{i}^{j}$ are calculated. Once the fuzzy membership function has been obtained, the de-fuzzified value can be calculated using the following centroid formula.

$$
\tilde{x}_{i}=\frac{\int \mu(z) \cdot z d z}{\int \mu(z) d z}
$$

These de-fuzzified values were obtained for all attributes and used to generate the simplified FCM as shown below in Figure 4. Such fuzzy cognitive maps were generated for all areas.

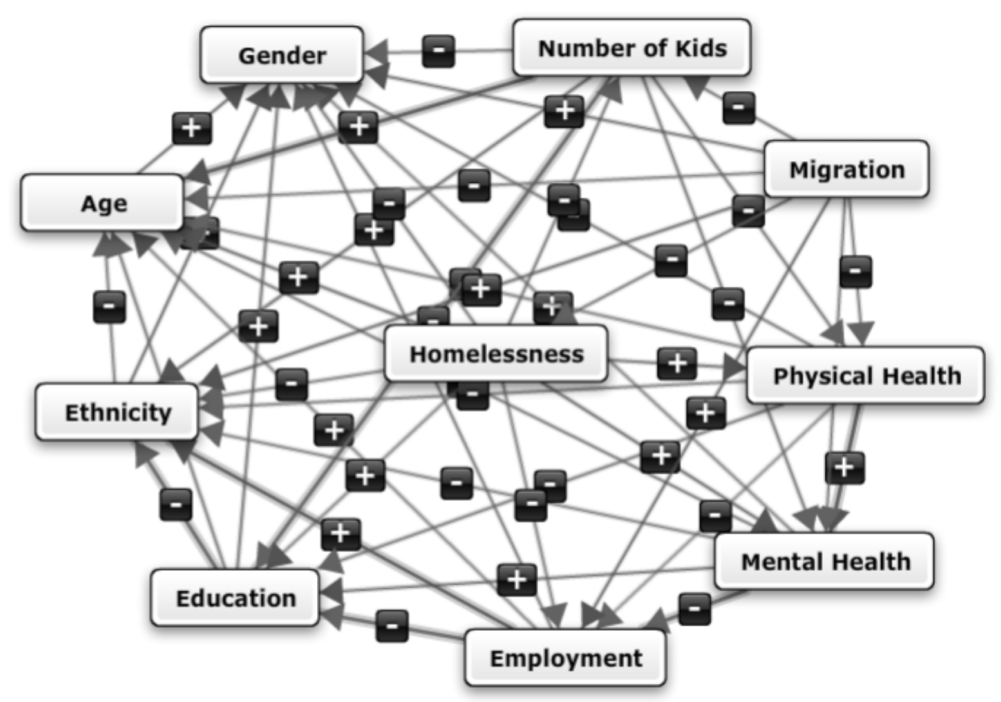

Figure 4: Simplified Fuzzy Cognitive Map generated during this study. 


\section{Analyses Based on the FCM Framework}

An important aspect of a study that can be performed using this framework is the sensitivity analysis, which determines how change in one variable affects other variables and vice versa. This framework can also be used to construct poverty index for a locality, which can be used to determine likelihood of a person becoming homeless based on several factors included in the FCM. Such indices were developed during a continuation of this study for five communities in northern Ontario, Canada (Sudbury, Timmins, Hearst, Moosonee, Cochrane) and are given below (see Naeem, 2020).

$$
\begin{aligned}
& h_{\text {Sudbury }}^{*}= 1.228288\left\{-p_{a g} w_{a g}+p_{g e} w_{g e}-p_{e t} w_{e t}+p_{e d} w_{e d}+p_{e m} w_{e m}+p_{m h} w_{m h}\right. \\
&\left.+p_{p h} w_{p h}-p_{n k} w_{n k}+p_{m i} w_{m i}-0.636202\right\}+1.0 \\
& h_{\text {Timmins }}^{*}=1.228123\left\{p_{a g} w_{a g}-p_{g e} w_{g e}-p_{e t} w_{e t}+p_{e d} w_{e d}+p_{e m} w_{e m}+p_{m h} w_{m h}-p_{p h} w_{p h}\right. \\
&\left.\quad-p_{n k} w_{n k}+p_{m i} w_{m i}-0.463766\right\}+1.0 \\
& h_{\text {Hearst }}^{*}=1.233076\left\{-p_{a g} w_{a g}+p_{g e} w_{g e}-p_{e t} w_{e t}+p_{e d} w_{e d}+p_{e m} w_{e m}-p_{m h} w_{m h}-p_{p h} w_{p h}\right. \\
&\left.\quad+p_{n k} w_{n k}+p_{m i} w_{m i}-0.515775\right\}+1.0 \\
& h_{\text {Moosonee }}^{*}=1.21748\left\{-p_{a g} w_{a g}-p_{g e} w_{g e}-p_{e t} w_{e t}+p_{e d} w_{e d}-p_{e m} w_{e m}+p_{m h} w_{m h}\right. \\
&\left.\quad+p_{p h} w_{p h}-p_{n k} w_{n k}+p_{m i} w_{m i}-0.306771\right\}+1.0 \\
& h_{\text {Cochrane }}^{*}=1.226751\left\{-p_{a g} w_{a g}+p_{g e} w_{g e}-p_{e t} w_{e t}+p_{e d} w_{e d}-p_{e m} w_{e m}+p_{m h} w_{m h}\right. \\
&\left.\quad-p_{p h} w_{p h}+p_{n k} w_{n k}+p_{m i} w_{m i}-0.456204\right\}+1.0
\end{aligned}
$$

The weights $(w)$ are as defined in Table 6 and the parameters $(p)$ are defined as in Table 7 below. The $(h)$ values have been normalized according to the following equation and values given in Table 9.

$$
h^{*}=\frac{1.0}{h_{\max }-h_{\min }}\left(h-h_{\max }\right)+1.0
$$


Table 6: Average normalized weights for different communities with standard deviations.

\begin{tabular}{|c|c|c|c|c|c|c|c|}
\hline & \multirow{2}{*}{$\begin{array}{c}\text { Weight } \\
\text { Parameter }\end{array}$} & \multicolumn{5}{|c|}{ Weight } & \multirow{2}{*}{$\begin{array}{c}\text { Spread } \\
\text { (Standard } \\
\text { Deviation) }\end{array}$} \\
\hline & & Sudbury & Timmins & Hearst & Moosonee & Cochrane & \\
\hline Age & $w_{a g}$ & 0.1075 & 0.1199 & 0.1029 & 0.1047 & 0.1075 & 0.0066 \\
\hline Gender & $w_{g e}$ & 0.1200 & 0.1089 & 0.1190 & 0.1076 & 0.1119 & 0.0057 \\
\hline Ethnicity & $w_{e t}$ & 0.0976 & 0.1037 & 0.1024 & 0.1042 & 0.1070 & 0.0034 \\
\hline Education & $w_{e d}$ & 0.1105 & 0.1163 & 0.1134 & 0.1190 & 0.1232 & 0.0049 \\
\hline Employment & $w_{e m}$ & 0.1105 & 0.1117 & 0.1134 & 0.1007 & 0.1046 & 0.0053 \\
\hline Mental Health & $w_{m h}$ & 0.1209 & 0.1117 & 0.1103 & 0.1222 & 0.1139 & 0.0054 \\
\hline $\begin{array}{l}\text { Physical } \\
\text { Health }\end{array}$ & $w_{p h}$ & 0.1234 & 0.1073 & 0.1071 & 0.1220 & 0.1081 & 0.0084 \\
\hline $\begin{array}{l}\text { Number of } \\
\text { Kids }\end{array}$ & $w_{n k}$ & 0.0991 & 0.1100 & 0.1190 & 0.1092 & 0.1119 & 0.0071 \\
\hline Migration & $w_{m i}$ & 0.1105 & 0.1106 & 0.1125 & 0.1103 & 0.1119 & 0.0010 \\
\hline
\end{tabular}

Table 7: Boundary values for homelessness index.

\begin{tabular}{|l|c|c|}
\cline { 2 - 3 } \multicolumn{1}{c|}{} & \multicolumn{2}{c|}{$h$} \\
\cline { 2 - 3 } \multicolumn{1}{c|}{} & Minimum & Maximum \\
\hline Sudbury & -0.177939 & 0.636202 \\
\hline Timmins & -0.350480 & 0.463766 \\
\hline Hearst & -0.295210 & 0.515775 \\
\hline Moosonee & -0.514600 & 0.306771 \\
\hline Cochrane & -0.358960 & 0.456204 \\
\hline
\end{tabular}

With these equations it was possible to compare results from different study areas with same parameters. To make such comparisons several hypothetical scenarios were generated depicting 
typical individuals; their homelessness index values were calculated and compared (see Figures 5 and 6). This indicated that there are spatially-dependent pathways to homelessness in Northern Ontario and a single set of criteria cannot be used to form policy to reduce homelessness in all of Northern Ontario.

\section{Discussion}

The Fuzzy Cognitive framework developed here can be effectively used to perform different types of analyses on poverty, homelessness and migration data, for example to develop index of poverty, as shown here. Although this framework was developed on the datasets obtained within the context of a multi-year community-university research conducted at Laurentian University, Canada that studied issues related to poverty, homelessness and migration in northeastern

Ontario, Canada (Kauppi et. al., 2015), the framework itself can be used on any similar dataset. The methodology thus developed is not unique to any specific dataset.

\section{References}

Dickerson J., Kosko B. (1994). Virtual worlds as fuzzy cognitive maps. Presence. 3(2), 173-189. Fowler, P.J., Hovmand, P.S., Marcal, K.E. and Das, S. (2019). Solving Homelessness from a Complex Systems Perspective: Insights for Prevention Responses. Annu. Rev. Public Health, 40:465-86.

Gray, S.A. et. al. (2013). Mental Modeler: A Fuzzy-Logic Cognitive Mapping Modeling Tool for Adaptive Environmental Management. 46th Hawaii International Conference on System Sciences. 
Hall, G. (2015). Pearson's Correlation Coefficient. Available at:

www.hep.ph.ic.ac.uk/ hallg/UG 2015/Pearsons.pdf.

Kauppi, C., Pallard, H., Faries, E. (2015). Poverty, Homelessness and Migration In Northeastern

Ontario, Canada. OIDA International Journal of Sustainable Development. Vol.8, No.4, pp.1122.

Naeem, R. (2020). A Study of Homelessness and Migration in Northern Rural and Urban

Centres in the Near North, Ontario, Canada, using GIS Techniques. PhD Thesis. Kingston

University, UK. 